\title{
TRANSPOSON-INDUCED NUCLEAR MUTATIONS THAT ALTER CHLOROPLAST GENE EXPRESSION.
}

\author{
ANNUAL REPORT \\ FOR THE PERIOD \\ 09/01/91-08/31/92
}

DE93 010774

\author{
DR. ALICE BARKAN, PRINCIPAL INVESTIGATOR \\ INSTITUTE OF MOLECULAR BIOLOGY \\ UNIVERSITY OF OREGON \\ EUGENE, OR 97403
}

\author{
PREPARED FOR THE \\ US DEPARTMENT OF ENERGY \\ DIVISION OF ENERGY BIOSCIENCES \\ OFFICE OF BASIC ENERGY SCIENCES \\ UNDER GRANT DE-FG06-91ER20054
}

\section{DISCLAIMER}

\begin{abstract}
This report was prepared as an account of work sponsored by an agency of the United States Government. Neither the United States Government nor any agency thereof, nor any of their employees, makes any warranty, express or implied, or assumes any legal liability or responsibility for the accuracy, completeness, or usefulness of any information, apparatus, product, or process disclosed, or represents that its use would not infringe privately owned rights. Reference herein to any specific commercial product, process, or service by trade name, trademark, manufacturer, or otherwise does not necessarily constitute or imply its endorsement, recommendation, or favoring by the United States Government or any agency thereof. The views and opinions of authors expressed herein do not necessarily state or reflect those of the United States Government or any agency thereof.
\end{abstract}


The long term goal of this project is to use mutant phenotypes as a guide to nuclear genes that determine the timing and localization of chloroplast development. The immediate goals of the funded grant proposal are to (1) identify nuclear mutants with defects in chloroplast gene expression from maize lines harboring active $M u$ transposons, (2) characterize their phenotypes to determine the precise defect in gene expression, (3) clone several of the most interesting mutations by exploiting the transposon tag, and (4) use the clones to further define the roles of these genes in modulating chloroplast gene expression.

In the original proposal, three mutants were described that had global defects in chloroplast gene expression. During the first year of funding, we have found that two of these mutations are allelic. Both alleles have global defects in chloroplast translation initiation, as revealed by the failure to assemble chloroplast mRNAs into polysomes. In addition, we have isolated and characterized three new mutants from $M u$ lines that have novel defects in chloroplast RNA metabolism. We are now ready to begin the task of cloning several of these genes, by using the $M u$ transposon tag. This work is outlined in detail below.

\section{A. ASSIGNMENT OF NEW GENE NOMENCLATURE.}

Until recently, we referred to all green, non-photosynthetic mutants as "high chlorophyll fluorescence" ( $h c f)$ mutants, because of their increased level of chlorophyll fluorescence. Because mutations of so many different loci with very different functions can result in this phenotype, this designation was not very informative. Therefore, we have now assigned new gene nomenclatures that reflect more completely the variety of mutant phenotypes.

We have grouped our various transposon-induced mutants into different classes according to the specific nature of the chloroplast defect. The assays used include Western analysis of the major chloroplast proteins, Northern analysis of chloroplast mRNAs, and analysis of chloroplast polysomes. Mutants that were already described in refereed publications were not renamed. Of relevance to this grant are the following two classes of mutants:

crp Mutants. crp mutants have unique defects in chloroplast RNA processing. One published mutant, $h c f 38$, also falls into this category (Barkan et al, 1986).

crpl - fails to accumulate monocistronic petB and petD mRNAs. Previously called hcf 36.

crp2 - fails to degrade excised group II introns. Previously called $h c f 143$.

crp3-fails to accumulate the predominant petA mRNA. Previously called $h c f l 42$.

cps Mutants. cps mutants have global defects in chloroplast protein synthesis, as revealed by a decrease in the levels of all thylakoid membrane complexes and RUBISCO, as well as a decreased association of all chloroplast mRNAs with polysomes. $h c f 7$ also falls into this category (Taylor et al., 1987).

cpsl-We have two alleles of this gene, cpsl-1 and cpsl-2. Previously called $h c f 129$ and $h c f 131$, respectively.

cps 2 - Previously called hcfl 33.

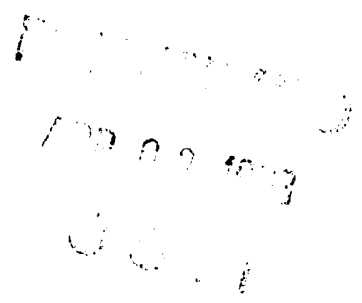




\section{B. PROGRESS DURING YEAR ONE.}

\section{Mutants Defective in Chloroplast Translation.}

Molecular Studies: Analysis of polysomes in mutant seedlings revealed that cps $1-1$, cps $I-2$, and cps 2 mutants have abnormally few ribosomes associated with most or all chloroplast mRNAs. Therefore, a global defect in translation initiation is likely to be the cause of the protein deficiencies in these mutants. We used these mutants together with $h c f 7$ (an EMS-induced mutant that also fails to assemble chloroplast polysomes), to assess whether decreased ribosome association might affect the stabilities of chloroplast mRNAs. While most chloroplast mRNAs accumulate to normal levels in these mutants, the abundance of the $r b c L$ mRNA is reduced fourfold in each case. These results suggest that a decreased association with ribosomes leads to the destabilization of this one mRNA. We speculate that changing association with ribosomes may play a role in the changing stability of this mRNA during normal chloroplast development. These and other studies of this set of mutants have been described in a manuscript that should be submitted for publication during 12/92.

Genetic Studies: Crosses between plants heterozygous for cps $1-1$ and $\operatorname{cps} 1-2$ yielded mutant progeny. Therefore, these two mutations are allelic. They are almost certainly distinct alleles because they do not arise from the same lineage, and $c p s I-2$ has a more severe translation defect than cps $1-1$.

Crosses between plants heterozygous for $h c f 7, \operatorname{cps} 2$, and $c p s l$ mutations, in ail pairwise combinations failed to yield any mutant progeny. Therefore these mutations represent three different genes.

Crosses with B-A translocation stocks revealed that $h c f 7$ maps to the long arm of chromosome 1, and cps 2 maps to the long arm of chromosome 6. Mapping crosses were also performed with cpsl mutants, but the progeny have not yet been analyzed.

\section{Mutants Defective in Chloroplast RNA Metabolism.}

Early in this funding period we identified three new mutants, with unique alterations in chloroplast mRNA metabolism.

crp 1: crpl mutants appear to be defective in a site-specific ribonuclease whose activity is required to generate translatable mRNAs from an inactive primary transcript.

We found that this mutant is non-photosynthetic due to the absence ( $<5 \%$ WT levels) of the cytochrome $\mathrm{f} / \mathrm{b} 6$ complex, and a reduction (10\% WT levels) in the PSI complex. Other chloroplast proteins accumulate normally. Examination of the mRNAs encoding missing proteins revealed the specific absence of the monocistronic transcripts encodingpet $B$ and petD (subunits of the cytochrome $\mathrm{s} / \mathrm{b} 6$ complex). These two genes lie adjacent to one another, and are transcribed as part of the $p s b B$ gene cluster. A tetracistronic primary transcript is ordinarily processed to yield tricistronic, dicistronic, and monocistronic transcripts derived from each gene in the transcription unit. The larger transcripts of this set of genes accumulate normally in crpl mutants, indicating that transcription itself is normal. The simultaneous loss of the monocistronc petB and petD transcripts is most easily explained by the failure of a site-specific ribonuclease to cleave the primary transcript between these two coding regions. Alternatively, this processing event may occur normally, but the processed transcripts may be very unstable in the absence of the crpl gene product. 
Despite an extensive search, we have not detected any other aberrant chloroplast transcripts in crpl mutants. Therefore, the CRP1 gene functions quite specifically in the metabolism of one set of chloroplast mRNAs. We are most intrigued by the fact that the loss of the monocistronic pet $B$ and petD transcripts correlates with the absence of the cytochrome $\mathrm{f} / \mathrm{b} 6 \mathrm{complex}$. Previously, the function of the extensive intercistronic RNA processing so common in chloroplasts has been mysterious. There has been no evidence that this processing was important for the ultimate expression of any chloroplast gene. The phenotype of $c r p l$ provides the first evidence that processing of a primary transcript to the monocistronic form is, at least in some cases, essential for efficient translation.

crp2: crp2 mutants over-accumulate excised group II introns or intron fragments, and may also be defective in translation elongation.

We found that the photosynthetic defect in this mutant is due to a 10 - fold reduction in the levels of all thylakoid complexes and of RUBISCO. This phenotype suggested a global defect in chloroplast gene expression. However, all chloroplast mRNAs examined were of normal size and abundance, and were associated with polysomes. We suspect therefore, that one aspect of the mutant phenotype is a decrease in the translation elongation rate.

The unique feature of the $c r p 2$ phenotype is that hybridization probes that include group II intron sequences from many different chloroplast genes reveal abundant, aberrant transcripts that are only barely detectable in WT samples. The sizes of these novel transcripts suggest that they are excised introns, or perhaps intron fragments. The single group I intron in higher plant chloroplasts did not accumulate detectably in either WT or crp2 chloroplasts.

We do not yet know the precise structure of the aberrant molecules. We would especially like to know whether they are branched molecules or lariats. This would enable us to evaluate the possibility that the mutant is defective in a lariat debranching enzyme. The relationship between this intron phenotype and the global loss of chloroplast proteins is also very intriguing: Do the excised introns poison the translation machinery? Or does the HCF143 gene product function both in intron degradation and in translation elongation? For example, the gene might encode an RNA helicase that is necessary for efficient translation, and also to dissociate excised introns from a splicing complex that would otherwise protect them from ribonucleases.

crp3: crp3 seedlings lack the predominant petA transcript, and therefore fail to synthesize or stabilize this mRNA.

The photosynthetic defect in this mutant is due to a 5-10 fold reduction in the levels of all thylakoid complexes and of RUBISCO. This phenotypeis similar to that observed in crp2 mutants, and suggested a global defect in chloroplast gene expression. However, most chloroplast mRNAs were of normal size and abundance, and were associated with polysomes. We suspect therefore, that this mutation may cause a decrease in the translation elongation rate (as we also suspect for crp2).

The novel feature of this mutant phenotype is the absence of the predominant transcript of the petA gene. No other deficiencies in chloroplast transcripts have been detected. We do not yet know whether this deficiency is due to a defect in transcription, or to decreased stability of this RNA. The relationship between the putative translation elongation defect and this altered mRNA metabolism remains obscure. 


\section{GOALS FOR YEAR TWO.}

We are most excited about the three mutants with defects in chloroplast RNA metabolism, crp1, crp2, and crp3. Mutants similar to these in any organism have not been described previously. We plan to complete the basic charactizations of these three mutants this year, and then prepare those results for publication. For example, we will determine whether the absence of the cytochrome $\mathrm{f} / \mathrm{b} 6$ complex in $\mathrm{crpl}$ is due to the failure to synthesize petD, petB, or both. This will enable us to focus on the relevant mRNAs in future studies of how processing to a monocistronic transcript facilitates translation. We will also complete an exhaustive analysis of chloroplast transcripts, to determine whether any other transcripts are improperly or incompletely processed.

Further characterization of the $\operatorname{crp} 2$ phenotype will include an exhaustive survey of the accumulation of all chloroplast introns. In addition, we will determine the structure of the intron/intron fragments that are overaccumulating, and test the prediction that this mutation causes a decrease in the rate of translation elongation.

We will then initiate the search for $M u$ transposons linked to each of these mutations. The crpl and crp 3 mutations are almost certainly due to insertions of $M u$ transposons, since tiny revertant (dark green) sectors are visible on pale green mutant leaves. Unfortunately, we have yet to observe revertant sectors on crp2 leaves. This may indicate that the mutation is not caused by $M u$, so we will proceed cautiously.

The translation mutants are less assured of defining novel genes: they may be defective in some previously described component of the translation machinery (e.g. a ribosomal protein or initiation factor). On the other hand, they could also define novel components of the translation machinery, or they could shed light on the functions of previously described components. All three mutants are very likely due to insertions of $M u$ transposons, since tiny revertant sectors are visible on pale green mutant leaves. Nonetheless, we do not plan to clone these genes at this time, since we have limited people-power, and are currently more excited about the RNA processing mutants. I plan to seek collaborators whose primary interests concern the chloroplast translation machinery; and who are interested in using the material we have generated to clone these genes. 

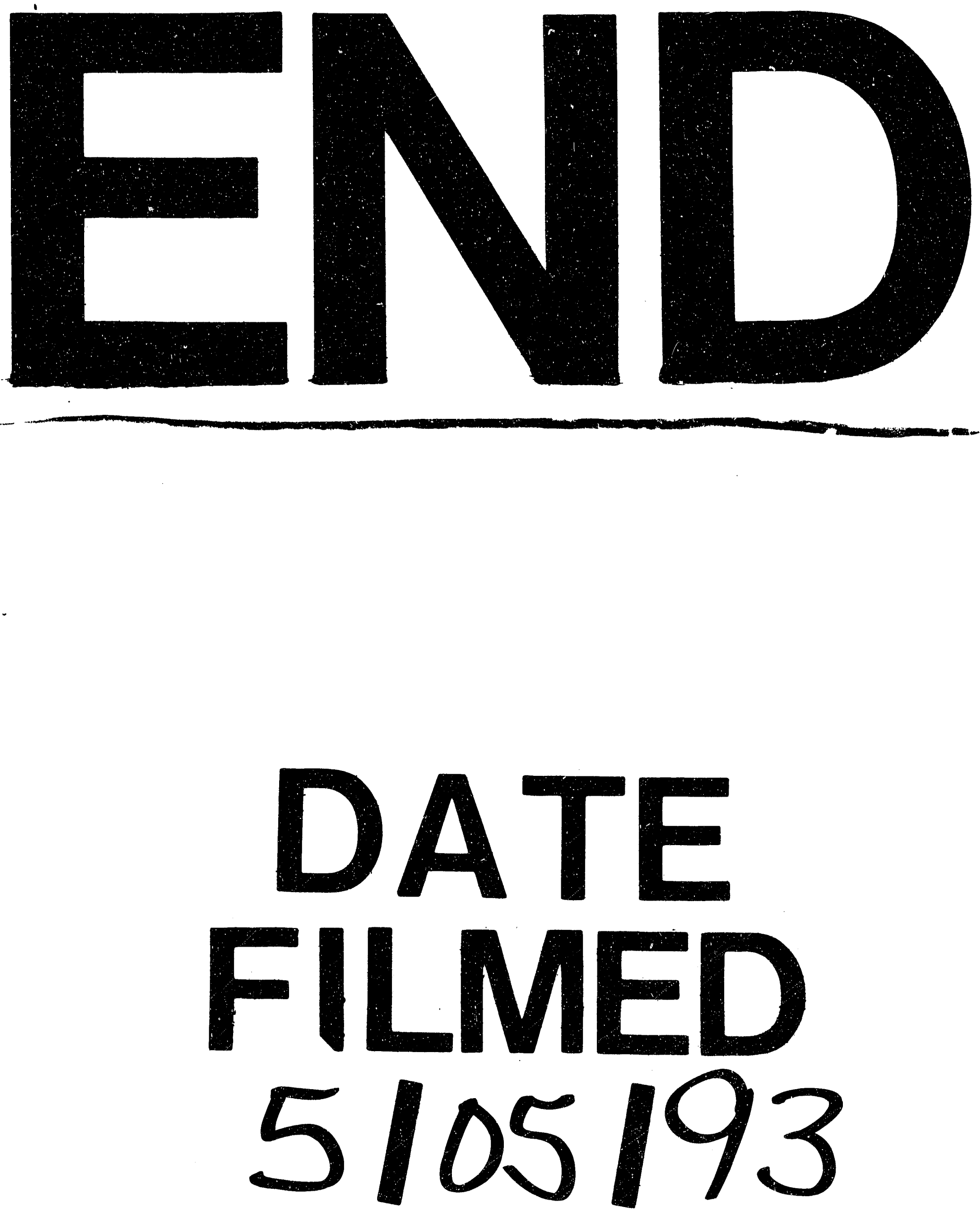


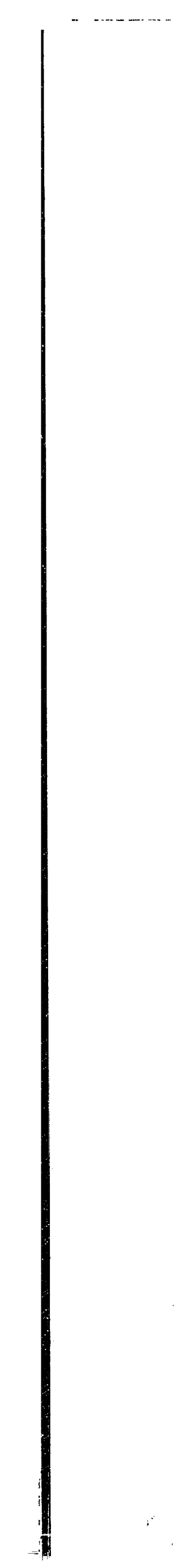

